\title{
Hiérarchies portuaires dans le monde \\ et changements régionaux de connectivité maritime, 1890-2010
}

César DUCRUET, CNRS

Bruno MARNOT, Université de la Rochelle

In : Buchet C. (dir), La mer dans l'Histoire / The Sea in History, Boydell \& Brewer Vol. 4, pp. 631-639.

\section{Introduction}

Le rôle du fait maritime dans l'évolution des sociétés humaines est difficile à évaluer faute d'informations précises sur l'importance même de l'activité maritime à l'échelle mondiale. Les statistiques de tonnage portuaire ne couvrent que partiellement le globe ou la période contemporaine. Le recours aux pavillons pour comparer la taille de la flotte des pays reste un pisaller eu égard aux immatriculations échappant aux contraintes fiscales. Les nombreux travaux faisant le point sur une région et/ou une période donnée sont difficilement synthétisables tant les sources utilisées diffèrent. L'une des rares solutions restantes est de considérer la façon dont ports, régions, ou nations sont reliés les uns aux autres par les flux du commerce maritime. Ceci est possible grâce à la redécouverte d'archives jusqu'ici inexploitées, celles de l'assureur maritime Lloyd's sur les mouvements de navires dans le monde, publiées depuis l'année 1696 de façon quotidienne ou hebdomadaire.

\section{Présentation de la source et résultats préliminaires}

La présente analyse propose ainsi de mesurer le nombre d'escales de navires par pays et couple de pays au niveau mondial à cinq dates-clé de la période contemporaine (1890, 1925, 1960, 1985 et 2008). Même si une part d'arbitraire peut apparaître dans leur choix et pourrait donner l'impression d'orienter l'étude, elles ont été choisies selon trois critères complémentaires : une certaine régularité de la fréquence, l'exclusion des séquences de grands conflits internationaux ou des crises économiques internationales et surtout des moments qui apparaissent historiquement signifiants : 1890 correspond au cœur de la mondialisation de la deuxième moitié du XIXe siècle, 1925 à une année «normale " d'échanges internationaux dans l'entre-deux-guerres, 1960 au cœur de la période de croissance mondiale des trente années d'après-guerre, 1985 au début de la mondialisation de la fin du XXe siècle et à la déstabilisation du bloc soviétique, enfin 2008 à la situation de la mondialisation au début du XXIe siècle, avant la crise financière des subprimes.

Le Shipping Index recense le dernier mouvement connu de chaque navire répertorié par Lloyd's, soit environ $80 \%$ de la flotte mondiale actuelle. Le nombre d'escales par pays et couple de 
pays est l'indicateur le plus comparable sur la période, même s'il a l'inconvénient de ne pas tenir compte de la taille des navires. En revanche, il exprime bien la fréquence de l'activité maritime et constitue par-là constitue un bon indicateur de puissance économique et logistique des Etats. Le découpage actuel des frontières étatiques permet de retracer l'évolution des pays sur la base d'un même territoire, en dépit des remodelages plus ou moins profonds ayant eu lieu.

Tout d'abord l'on s'intéresse au réseau maritime mondial des flux entre pays et à sa structure d'ensemble, qui par le recours à quelques indicateurs synthétiques nous renseigne beaucoup sur la logique globale des échanges (Tableau 1). De façon logique, le nombre de pays et de navires a cru très régulièrement sur toute la période, reflétant ainsi l'augmentation continuelle des partenaires commerciaux et de la flotte mondiale, tous trafics confondus. Le nombre total d'escales dans le monde reflète avant tout des périodes de croissance et de crise. Or si les impacts respectifs de la Seconde Guerre Mondiale (1946-1951) ou des chocs pétroliers (1975-1980) étaient attendus, le déclin du nombre d'escales à partir de 1995 (on retrouve en 2008 un niveau inférieur à 1925), lui, s'explique davantage par la rationalisation des escales par les compagnies maritimes. En effet, le nombre moyen d'escales par navire a considérablement chuté dans la période récente, passant d'environ 1 en 1890 à 0,5 en 2008.

D'autres tendances opposent nettement les périodes d'avant et après 1970, comme la baisse de la concentration du nombre d'escales entre les pays (Gini, HHI), ce qui est dû à l'expansion géographique du commerce maritime. En revanche, si le réseau se consolide, eu égard à l'augmentation du nombre de liens inter-pays jusque vers 1990, on observe comme pour les escales une baisse récente depuis 1995, elle aussi due à la rationalisation des escales, ce qui se reflète aussi dans la baisse récente du nombre maximum et moyen de liaisons dont un pays peut bénéficier (degré). Le maillage du réseau (excentricité) s'est renforcé, permettant des liens plus directs entre pays, tandis que la hiérarchisation du réseau (loi de puissance) a augmenté fortement et très rapidement en fin de période (1995-2008), retrouvant alors un niveau initial. Ainsi, face à la croissance des échanges, le réseau maritime s'est densifié mais en même temps a connu une centralisation et rationalisation récente qui rappelle la configuration d'il y a plus d'un siècle.

La cartographie du nombre d'escales par pays aux deux années extrêmes de la période d'étude montre bien en quoi l'évolution du réseau fut également marquée par d'important changements géographiques (Figure 1). Ce glissement de l'Atlantique au Pacifique est bien l'une des causes des tendances lourdes observées: l'allongement des distances parcourues a accentué la nécessité de centraliser les trafics en certains points stratégiques du globe. L'une des stratégies des acteurs maritimes mais aussi des Etats a donc été de se spécialiser dans le trafic de transit, une façon de ces derniers de garder la mainmise sur l'architecture mondiale des flux non seulement physiques mais informationnels. 


\section{Evolution diachronique générale (1890-2008)}

L'analyse qui suit fournit tout d'abord un regard nouveau sur l'évolution de la hiérarchie maritime mondiale (Tableau 2), à partir des vingt premiers pays par le nombre d'escale. Ensuite, le réseau maritime simplifié (flux dominants) révèle le niveau de centralisation des flux maritimes entre pays du monde (Figure 2).

L'analyse en première approximation du tableau 2 montre que la part des vingt premiers pays régresse de façon substantielle sur un peu plus d'un siècle, passant de presque $82 \%$ du nombre d'escales mondiales en 1890 à presque $62 \%$ en 2008. Cette évolution peut être interprétée comme l'approfondissement du processus séculaire de multilatéralisation des échanges, mais aussi par le nombre croissant de pays dont les ports participent au processus de mondialisation (multiplication des escales, des hubs et des spokes). Sur la longue durée, la multilatéralisation des échanges prend la configuration d'une structure réticulaire de plus en plus éclatée et complexe en raison de la multiplication et de la ramification des sous-réseaux qui s'articulent aux flux dominants. En outre, multilatéralisation et tendance à l'éclatement du schéma réticulaire s'accompagnent d'un rééquilibrage Nord-Sud au sommet de la hiérarchie des pays comptabilisant le plus grand nombre d'escales mondiales. De l'analyse combinée du tableau et des graphes décrivant les liens de dépendances des flux majeurs se dégagent trois séquences chronologiques majeures.

\section{Domination britannique et " bipôle atlantique »}

La première séquence (années 1890 et 1925) se caractérise par la domination du RoyaumeUni comme centre des échanges maritimes mondiaux à un moment, du reste, où elle a cessé d'être la première puissance industrielle. Toutefois, elle demeure durant cette période la première puissance commerciale, la première flotte marchande et se trouve à la tête d'un empire colonial qui atteint son apogée dans l'entre-deux-guerres. La suprématie commerciale de la Grande-Bretagne apparaît en l'occurrence par le nombre et la diversité géographique de ses liens, ce qui confirme le degré d'extraversion et de mondialisation de son économie (Saul, 1960). Les neuf premiers ports britanniques se caractérisent par leurs horizons mondiaux, à commencer par les deux plus puissants d'entre eux, Londres et Liverpool (Starkey, 1999). En 1890, trois directions majeures s'articulent autour du centre britannique. Des Etats d'Amérique du Sud, comme l'Argentine, le Brésil, voire le Chili, avec prolongement vers les îles caribéennes, apparaissent comme des sous-pôles importants. Leur forte intégration commerciale - et financière - à la Grande-Bretagne a servi d'argument pour considérer ces pays comme faisant partie de "l'empire informel » britannique. La deuxième catégorie de liens commerciaux majeurs est constituée par les "colonies de peuplement » britannique, en premier lieu l'Australie, et, à moindre titre, le Canada, exportateurs essentiels, au 
même titre que les pays d'Amérique du Sud, de matières premières et de produits agricoles en direction de la métropole britannique.

Cependant, le troisième lien commercial majeur entre Royaume-Uni et Etats-Unis est de loin le plus intense. La figure 1 est la matérialisation cartographique du "bipôle nord-atlantique » (J. Guillaume, 1998), qui se caractérise par un volume d'échanges élevé et diversifiés (matières premières, biens manufacturés, migrants) le long de la route maritime qui s'est imposée comme l'axe majeur de la mondialisation du XIXe siècle entre la première puissance économique mondiale et la république américaine en passe de devenir la première puissance industrielle mondiale. Ce bipôle est notamment matérialisé par la ligne transatlantique Liverpool-New York. Le port de la côte Nord-Est des Etats-Unis s'est alors affirmé comme la principale porte d'entrée et de sortie du commerce extérieur américain avec un avant-pays de niveau mondial (Heffer, 1986). L'Allemagne, première puissance industrielle européenne et puissance commerciale grandissante, appartient au réseau des flux majeurs de ce bipôle par le biais de ses relations commerciales importantes avec les Etats-Unis. Ce n'est pas le cas de la France, quatrième puissance économique mondiale, dont les liens commerciaux majeurs se concentrent sur l'Europe de l'Ouest et son empire colonial africain.

En 1925, 7 des 20 premiers pays en matière d'escales appartiennent au monde occidental (Etats-Unis et Europe de l'Ouest). S'affirme donc une concentration des flux dans le centre de l'économie mondiale. La position de l'Europe de l'Ouest s'est considérablement renforcée et joue un rôle accru dans l'animation des échanges maritimes internationaux qui se reconstituent après la Première Guerre mondiale. La promotion de la Belgique (Anvers) et de la Hollande (Rotterdam) s'explique par leur rôle désormais indiscuté de portes d'entrée et de sortie de l'Europe. Tout aussi notables sont les promotions des pays du Nord de l'Europe (Norvège, Suède, Danemark) qui depuis la fin du XIXe siècle ont (re)constitué de puissantes flottes marchandes, ainsi que celle du Japon, qui s'affirme comme la seule grande puissance industrielle non-européenne, aux visées impérialistes régionales.

La reconstitution des liens commerciaux internationaux de l'après-guerre révèle la prééminence du Royaume-Uni, dont la position est plus que jamais centrale. Les ports britanniques totalisent $20 \%$ du total des escales mondiales et la Grande-Bretagne apparaît bien comme le moyeu d'une roue composée de plusieurs rayons saillants. On assiste, par rapport à 1890, à une évolution vers une structure monocentrée sur la Grande-Bretagne, tandis que les connections du pôle américain se réorientent dans deux directions majeures : l'«arrière-cour » des Caraïbes et de l'Amérique centrale, d'une part, et les relations transpacifiques, d'autre part, avec l'intensification des liens commerciaux avec le Japon. A la jonction de ces deux espaces se trouve Panama, dont le canal ouvert en 1914 est sous contrôle américain. Si les relations entre Royaume-Uni et Etats-Unis apparaissent toujours comme le lien le plus important, le pôle américain a donc relativement perdu 
de son importance par rapport aux connexions entre le Royaume-Uni et l'Europe, d'une part, et entre la métropole britannique et son empire colonial, d'autre part. L'interdépendance croissante de l'économie britannique avec son empire annonce le système de préférence impériale formalisé dans les années 1930 (accords d'Ottawa en 1932). II repose notamment sur les liens accrus avec les dominions, notamment l'Australie et l'Afrique du Sud, et l'Inde, qui joue toujours le rôle de clé de voûte du système de la balance des paiements britanniques.

\section{La polarisation américaine}

L'émergence de la nouvelle superpuissance dominant le bloc occidental et libéral dans les quarante années qui suivent la fin de la Deuxième Guerre mondiale se traduit par la substitution des Etats-Unis au Royaume-Uni comme centre nerveux des échanges internationaux où la maritimisation s'amplifie constamment. Comme pour le Royaume-Uni, il existe une corrélation apparente entre puissance politique / rayonnement international/ puissance commerciale. Les relations transatlantiques entre les Etats-Unis et l'Europe se matérialisent par la multiplication de sous-pôles (France, Allemagne, Italie), dont la Grande-Bretagne n'est que la composante la plus importante. Ces liens décrivent le rôle des Etats-Unis dans le relèvement économique de l'Europe de l'Ouest et les partenariats commerciaux étroits entre les deux composantes de I'OTAN. Quant au Royaume-Uni, la disparition de son empire réduit ses connexions majeures à ses anciennes colonies de peuplement dans le cadre du Commonwealth (Australie, Nouvelle-Zélande, Canada). En revanche, les Etats-Unis se sont substitués à l'Angleterre dans les flux majeurs avec l'Inde indépendante, qui est entrée dans l'orbite stratégique américaine). Malgré son déclassement économique dans la hiérarchie mondiale, la Grande-Bretagne se caractérise toujours comme un pôle majeur, avec un nombre et une intensité des relations commerciales majeures très élevés.

La montée en puissance des liens entre Etats-Unis et Japon constatée en 1925 est confirmée en 1960. Elle s'inscrit désormais dans le cadre d'un partenariat étroit entre les deux pays au sein duquel la superpuissance américaine joue le rôle de tuteur économique et de protecteur militaire de son ancien adversaire vaincu en 1945. L'intensité de ces liens commerciaux témoigne du rôle joué par les Etats-Unis dans le relèvement économique de leur ancien ennemi du Pacifique. La puissance commerciale et industrielle nippone s'affirme par l'édification d'une mégalopole portuaire constituée des trois ensembles Tokyo-Yokohama, Osaka-Kobé et Kitakyushu. Elle est le produit d'une politique ambitieuse de poldérisation et de construction d'îles-ports artificielles loties de vastes zones industrialo-portuaires qui s'imposent comme les poumons économiques du pays. Pays le plus développé d'Extrême-Orient, le Japon s'affirme d'ailleurs comme le noyau d'un sous-pôle régional avec des liens majeurs avec la Chine communiste et Singapour. Au fait de leur puissance, les EtatsUnis se trouvent au cœur des relations transpacifiques et transatlantiques. Si le graphe rappelle à 
certains égards la configuration de la fameuse "triade " (Amérique du Nord-Europe de l'OuestJapon), l'intérêt d'une telle représentation est de substituer à la neutralité géo-commerciale de cette notion sa réalité géopolitique, à savoir une structure tripolaire centrée sur le leader du monde occidental et libéral, qui inonde les marchés de consommation de ses alliés en biens manufacturés et culturels.

Cependant le tableau et le graphe de l'année 1960 révèlent d'autres évolutions non moins significatives. Le rôle des pays fournisseurs de matières premières (Inde, Chine, Australie, Argentine, Brésil) de la mondialisation du XIXe siècle n’a pas disparu mais a relativement diminué. Si la route par I'Afrique du Sud a perdu de son importance, le nombre de ports touchés sur la route Golfe d'Omanmer Rouge-Suez-Méditerranée a sensiblement augmenté en raison de l'essor du trafic des hydrocarbures. L'apparition de l'URSS parmi les 20 pays comptant le plus d'escales mérite également d'être signalée. Elle correspond à l'essor de ses relations maritimes avec les pays extra-européens relevant de sa sphère d'influence politique et économique. C'est le tournant décrit par A. Vigarié (1995) sous l'expression de "maritimisation des socialismes » où le commerce maritime devient un moyen d'intervention extérieure, auprès des pays du tiers-monde notamment; sans oublier le développement d'échanges avec les pays capitalistes même s'il demeure marginal dans le commerce extérieur de l'URSS.

\section{Vers la dispersion multipolaire}

L'actuelle mondialisation qui s'est esquissée à partir des années 1980 et s'est renforcée avec l'implosion du bloc communiste au début des années 1990 ne se traduit pas par un renforcement de la centralité américaine. Les graphes relatifs aux 1985 et 2008 se caractérisent par une tendance croissante à l'éclatement multipolaire de la structure des échanges maritimes. Contrairement aux phases antérieures, il n'apparaît plus d’Etat dominant dans le nombre et la diversité géographique des relations.

Le tableau montre que si les Etats-Unis demeurent, comme en 1960, le premier pays au monde pour le nombre d'escales, celui-ci a déjà diminué en valeur absolue de $20 \%$ en 1985 . Le Japon se hisse à la deuxième place pour le nombre d'escales, ce qui confirme son statut d'économie la plus dynamique du monde dans les années 1980 et de modèle pour les "dragons asiatiques ». De surcroît, La Chine et le port franc de Singapour, qui constitue depuis le XIXe siècle la porte d'entrée et de sortie de toute l'Asie du Sud-Est, se hissent aux premières places des Etats concentrant le plus d'escales. La nouvelle hiérarchie traduit un processus de déplacement des courants d'échanges internationaux et du centre de gravité du commerce mondial. En contrepoint, le poids de l'Europe de l'Ouest diminue relativement dans l'animation du commerce maritime, au profit des pays de l'Europe méditerranéenne (Espagne, Italie, Grèce), tandis que se confirme la place nouvelle de l'URSS - en 
l'occurrence Russie et Ukraine avec son grand complexe portuaire polyfonctionnel d'Odessa - et le rôle croissant de l'Arabie Saoudite et des monarchies pétrolières.

La représentation graphique des liens de dépendance majeurs révèle l'existence d'une structure tripolaire avec un bipôle Etats-Unis-Japon, un axe asiatique centré sur Singapour et un troisième sur l'Europe de l'Ouest. Le premier ensemble témoigne d'une évolution historiquement très significative, à savoir la disparition de la connexion séculaire majeure entre les Etats-Unis et le Royaume-Uni. Elle confirme une évolution en germe dans la représentation graphique de 1960. En 1985, les liens majeurs Etats-Unis-Europe de l'Ouest s'effectuent par l'Italie qui s'affirme comme le centre d'un pôle régional Méditerranée-Mer Noire et l'espace de mise en contact commercial du monde libéral avec certains pays du giron communiste (Cuba, Vietnam, Ukraine, Bulgarie). La distension des liens entre les Etats-Unis et les principales puissances économiques de l'Europe de l'Ouest est contrebalancée par la prépondérance des escales avec le Japon, ce qui confirme le tropisme asiatique et pacifique de la puissance américaine. Le rayonnement du pôle nippon s'élargit de l'Extrême-Orient - où il perd certaines connexions majeures comme la Chine et Singapour - à l'Océanie et à l'Océan Indien.

Par rapport à 1960, un ensemble extrême-oriental s'est individualisé, centré sur le hub singapourien qui rayonne sur le sud-est asiatique, le monde indien et la Chine. Pour contrarier l'influence de l'URSS sur l'aire d'obédience communiste, la Chine entre dans un processus de maritimisation active en développant notamment des flux avec deux pays européens communistes, la Roumanie et l'Albanie, ce dernier Etat étant dissident du bloc soviétique depuis 1960 pour se rapprocher de la République populaire de Chine.

L'ensemble européen s'organise autour des trois premières puissances économiques de la CEE que sont le Royaume-Uni, la République Fédérale d'Allemagne et, à moindre niveau la France. Cet ensemble montre le rôle d'intégration intra-européen du Marché Commun. Le premier constat significatif est la régression britannique dont les flux majeurs se sont géographiquement réduits et "(ré)européanisés " après une très longue séquence mondiale. Le port de Rotterdam, alors considéré comme le premier port mondial en termes de tonnage manutentionné, représente la principale porte d'entrée de la Grande-Bretagne en Europe. Cependant, malgré la suprématie de leurs établissements portuaires, ni la Hollande ni la Belgique ne constituent des sous-pôles maritimes, en raison de l'importance de leur fonction de redistribution continentale. Le sous pôle allemand se caractérise par l'importance des flux avec le nord de l'Europe et l'Europe de l'Est soviétique (URSS qui inclut les pays Baltes, Pologne). On peut y voir la traduction commerciale de l'Ostpolitik, c'est-à-dire de la politique de normalisation des relations avec l'Europe de l'Est lancée par le chancelier Willy Brandt. Du côté français, se vérifie la constance historique du flux avec les 
pays de son ancien domaine colonial et avec le Benelux, porte d'entrée de produits vers la France, d'un côté, et de sortie des produits français, de l'autre.

En 2008, le bipôle Etats-Unis-Japon et l'ensemble Extrême-Orient se sont rassemblés par la liaison Chine-Japon. Mais le nouvel axe nerveux relie la Chine à Singapour. La Chine occupe la première place pour le nombre d'escale, reflet de son nouveau statut $d^{\prime}$ " atelier du monde » : les plus grands armements mondiaux viennent y remplir leurs porte-conteneurs, mais la Chine affiche aussi l'ambition politique de se doter d'une puissante marine marchande, doublée d'une volonté d'influence politique régionale non moins évidente. Du reste, la nouvelle hiérarchie des 20 pays cumulant le plus grand nombre d'escales révèle un bouleversement historique majeur de l'époque contemporaine : 3 pays d'Extrême-Orient (et 6 dans le top 20) figurent dans les 5 premiers contre un seul pays Européen, le Royaume-Uni, en raison de son rang économique et de son caractère insulaire. Le tableau souligne également le nouveau rang occupé par les économies émergentes dans la structuration du commerce maritime international (Brésil, Russie, Inde, Chine).

La Chine s'affirme comme le centre d'un quadripôle qui confirme la dimension nouvelle des relations commerciales Amérique du Nord-Asie-Pacifique. La Chine ne constitue toutefois pas le hub d'un pôle étoffé de rayonnement mondial, comme ce fut le cas pour le Royaume-Uni et les Etats-Unis au temps de leur hégémonie. C'est en fait Singapour qui joue davantage ce rôle, mais sans bénéficier de la puissance politique. La Cité-Etat de la péninsule malaise constitue le grand carrefour sudasiatique autour duquel rayonnent des connexions majeures avec l'Inde, l'Amérique du Sud, le continent africain via l'Afrique du Sud -sous-pôle régional qui s'étoffe - et les Emirats Arabes Unis se sont substitués à l'Arabie Saoudite comme plaque tournante de l'économie du golfe persique. Au sein de cette structure, les Etats-Unis se trouvent en position excentrée. De façon paradoxale, Ils n'ont plus la densité spatiale des liens commerciaux correspondant à leur rôle d'hyperpuissance.

L'Europe se trouve à l'écart des flux majeurs de la structure centrale et se dédouble même en trois ensembles très distincts : l'un méditerranéen centré sur l'Espagne, avec la France, l'Italie et la Grèce; I'autre nord-européen (Royaume-Uni, Hollande, Belgique, Allemagne) et un ensemble oriental Russie-Turquie qui structure un ensemble composé de partenaires commerciaux historiques (Ukraine, Egypte, Jordanie, Syrie, Bulgarie).

\section{Conclusion}

L'évolution contemporaine du réseau maritime mondial fournit de nombreuses réponses quant au rôle changeant des Etats dans l'articulation physique des échanges internationaux. Si à la fin du XIXème siècle, le niveau de trafic reflète bel et bien la puissance commerciale et donc le volume des échanges, la fin du XXème siècle est animée de logiques plus complexes. D'un côté, la rationalisation et concentration des escales de navires en certains points stratégiques pour le trafic 
de transit a donné à certains pays un niveau de trafic bien supérieur à la demande commerciale. De l'autre, de grands partenaires comme la Chine restent finalement peu centraux dans le réseau malgré le volume de leurs échanges. Dans ce cas précis, le facteur politique joue bien plus que le facteur économique et technique, le réseau asiatique restant centralisé par la Corée du Sud, Hong Kong, Taiwan et Singapour, même si la Chine joue commercialement un rôle qui va en s'amplifiant. En Europe, les pays du Benelux s'imposent comme portes d'entrée principales du continent. Cette spécialisation accompagne et accentue la mainmise sur l'information logistique (et par-là sécuritaire, géostratégique) par quelques grandes plates-formes mondiales toujours plus en avance sur le plan technologique de la manutention et des échanges de données informatisées.

\section{Bibliographie}

GuillaUme, Jacques, "Les ports de commerce entre ambiance atlantique et mondialisation ", Historiens et Géographes, n 363, "L'Atlantique, un regard géographique ", août-septembre 1998, p. 199-205.

Heffer, Jean, Le Port de New York et le commerce extérieur américain, 1860-1900, Paris, Publications de la Sorbonne, 1986.

Miller Michael B. (2012), Europe and the Maritime World. A Twentieth-Century History, Cambridge, Cambridge University Press.

SAUL Samuel B., Studies in British Overseas Trade, 1870-1914, Liverpool, Liverpool University Press, 1960.

StARKEY, David J. (ed.) (1999), Shipping Movements in the Ports of the United Kingdom, 1871-1913. A Statistical Profile, Exeter, University of Exeter Press.

VIGARIE André (1995), La mer et la géostratégie des nations, Paris, Economica. 


\begin{tabular}{|c|c|c|c|c|c|c|c|c|c|c|c|c|}
\hline $\begin{array}{l}\frac{\bar{\pi}}{2} \\
\stackrel{0}{\|}\end{array}$ & 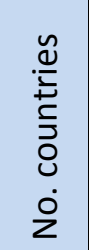 & $\begin{array}{l}\frac{n}{N} \\
\breve{N} \\
\stackrel{0}{0} \\
\dot{0}\end{array}$ & 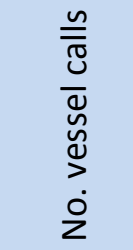 & 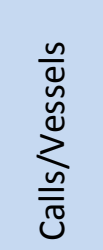 & 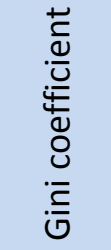 & 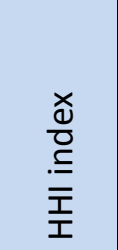 & $\begin{array}{l}\frac{\tilde{v}}{\underline{I}} \\
\dot{0} \\
z\end{array}$ & 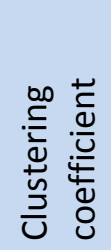 & 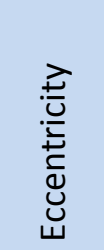 & 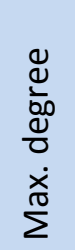 & 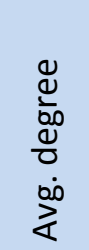 & 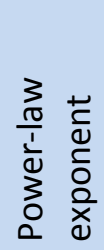 \\
\hline 1890 & 122 & 8,781 & 17,950 & 1.023 & 0.823 & 0.080 & 749 & 0.621 & 0.472 & 94 & 12.3 & 0.753 \\
\hline 1925 & 150 & 12,460 & 0,704 & 1.621 & 0.832 & 0.075 & 1,333 & 0.676 & 0.481 & 114 & 17.8 & 0.627 \\
\hline 1930 & 150 & 12,144 & 2,154 & 1.730 & 0.825 & 0.075 & 1,417 & 0.671 & .535 & & 18.9 & 0.601 \\
\hline 1935 & 146 & 11,236 & 38,836 & 1.772 & 0.809 & 0.072 & 1,468 & 0.650 & 0.574 & 104 & 20.1 & 0.579 \\
\hline 1940 & 145 & 11,651 & 9,162 & 1.767 & 0.811 & 0.080 & 1,454 & 0.758 & 0.500 & 102 & 20.0 & 577 \\
\hline 1946 & 152 & 14,736 & 32,448 & 1.118 & 0.801 & 0.077 & 1,291 & 0.602 & 0.481 & 109 & 17.0 & 0.677 \\
\hline 1951 & 149 & 11,815 & 2,088 & 1.390 & 0.779 & 0.054 & 1,557 & 0.663 & 533 & 107 & 20.9 & .562 \\
\hline 1960 & 168 & 14,317 & 42,698 & 1.525 & 0.770 & 0.046 & 2,101 & 0.665 & 0.632 & 122 & 25.0 & 0.553 \\
\hline 1965 & 166 & 16,265 & 58,044 & 1.830 & 0.784 & 0.058 & 2,364 & 0.667 & 0.596 & 129 & 28.5 & 0.492 \\
\hline 1970 & 169 & 15,948 & 45,412 & 1.412 & 0.741 & 0.039 & 2,568 & 0.642 & 0.612 & 129 & 30.4 & 0.442 \\
\hline 1975 & 164 & 20,115 & 43,848 & 1.068 & 0.735 & 0.034 & 2,474 & 0.660 & 0.615 & 123 & 30.2 & 0.437 \\
\hline 1980 & 166 & 21,066 & 40,446 & 0.953 & 0.731 & 0.031 & 2,505 & 0.660 & 0.647 & 126 & 30.2 & 0.441 \\
\hline 1985 & 173 & 22,942 & 44,034 & 0.927 & 0.741 & 0.030 & 2,632 & 0.619 & 0.651 & 125 & 30.4 & 0.460 \\
\hline 1990 & 173 & 23,806 & 57,456 & 1.142 & 0.753 & 0.031 & 3,091 & 0.662 & 0.637 & 136 & 35.7 & 0.341 \\
\hline 1995 & 175 & 21,423 & 46,482 & 1.022 & 0.743 & 0.028 & 2,864 & 0.651 & 0.642 & 127 & 32.7 & 0.388 \\
\hline 2000 & 182 & 22,162 & 42,552 & 0.875 & 0.764 & 0.030 & 2,325 & 0.580 & 0.670 & 113 & 25.6 & 0.561 \\
\hline 2008 & 177 & 25,212 & 28,564 & 0.518 & 0.752 & 0.027 & 1,680 & 0.522 & 0.595 & 92 & 19.0 & 0.695 \\
\hline
\end{tabular}

Tableau 1 : Evolution du réseau maritime mondial, 1890-2008 


\begin{tabular}{|c|c|c|c|c|c|c|c|c|c|c|}
\hline \multirow{2}{*}{ Rank } & \multicolumn{2}{|c|}{1890} & \multicolumn{2}{|c|}{1925} & \multicolumn{2}{|c|}{1960} & \multicolumn{2}{|c|}{1985} & \multicolumn{2}{|c|}{2008} \\
\hline & Country & Calls & Country & Calls & Country & Calls & Country & Calls & Country & Calls \\
\hline 1 & U.K. & 7483 & U.K. & 15036 & U.S.A. & 10231 & U.S.A. & 8542 & China & 5401 \\
\hline 2 & U.S.A. & 5654 & U.S.A. & 8799 & U.K. & 8890 & Japan & 7597 & U.S.A. & 4742 \\
\hline 3 & Argentina & 3317 & Germany & 3796 & Japan & 4232 & U.K. & 5172 & Singapore & 4448 \\
\hline 4 & Brazil & 1721 & France & 3408 & Germany & 4170 & Germany & 4348 & Japan & 3852 \\
\hline 5 & France & 1594 & Italy & 2803 & Netherlands & 2992 & Italy & 3757 & U.K. & 2780 \\
\hline 6 & Barbados & 1588 & Belgium & 2758 & Canada & 2747 & Netherlands & 3434 & Netherlands & 2528 \\
\hline 7 & Australia & 1464 & Netherlands & 2467 & Sweden & 2398 & China & 3417 & Spain & 2479 \\
\hline 8 & Uruguay & 1154 & Argentina & 2456 & Italy & 2328 & Spain & 2774 & Italy & 2454 \\
\hline 9 & Spain & 1144 & Australia & 2270 & France & 2263 & France & 2633 & Brazil & 2400 \\
\hline 10 & Germany & 1059 & Spain & 1888 & India & 1868 & Singapore & 2480 & Russia & 2359 \\
\hline 11 & Chile & 1052 & India & 1588 & Belgium & 1801 & Australia & 2337 & Turkey & 2216 \\
\hline 12 & South Africa & 1019 & Norway & 1548 & Australia & 1671 & Brazil & 2189 & U.A.E. & 2140 \\
\hline 13 & Italy & 706 & Japan & 1461 & Finland & 1482 & Belgium & 2158 & Australia & 2037 \\
\hline 14 & Canada & 613 & Sweden & 1245 & Argentina & 1372 & India & 1964 & India & 1986 \\
\hline 15 & Portugal & 478 & Brazil & 1116 & Norway & 1319 & Greece & 1948 & South Korea & 1935 \\
\hline 16 & India & 471 & South Africa & 1050 & Spain & 1302 & Russia & 1706 & France & 1611 \\
\hline 17 & China & 391 & Denmark & 1049 & China & 1198 & Saudi Arabia & 1655 & Indonesia & 1508 \\
\hline 18 & Indonesia & 375 & China & 996 & Denmark & 1023 & Canada & 1577 & Germany & 1483 \\
\hline 19 & Netherlands & 340 & Canada & 904 & Brazil & 995 & Argentina & 1508 & Belgium & 1412 \\
\hline \multirow[t]{3}{*}{20} & Trinidad & 334 & Chile & 764 & Russia & 968 & Ukraine & 1442 & Hong Kong & 1329 \\
\hline & World & 39077 & & 73103 & & 82143 & & 101626 & & 82629 \\
\hline & Top 20 (\%) & 81.8 & & 78.5 & & 67.3 & & 61.6 & & 61.8 \\
\hline
\end{tabular}

Tableau 2 : Vingt premiers pays par le nombre d'escales, 1890-2008 


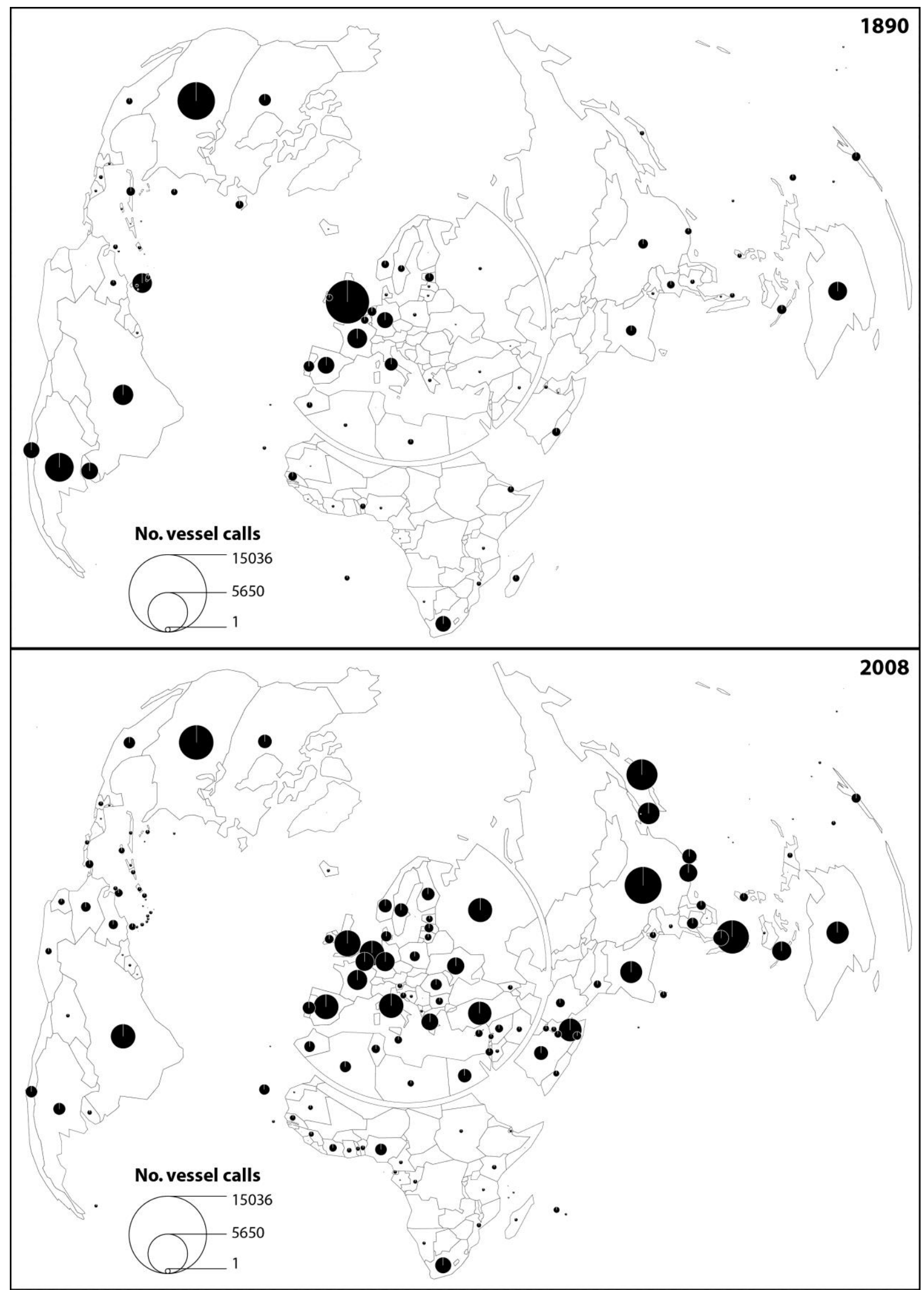

Figure 2 : Nombre d'escales de navires par pays, 1890 et 2008 


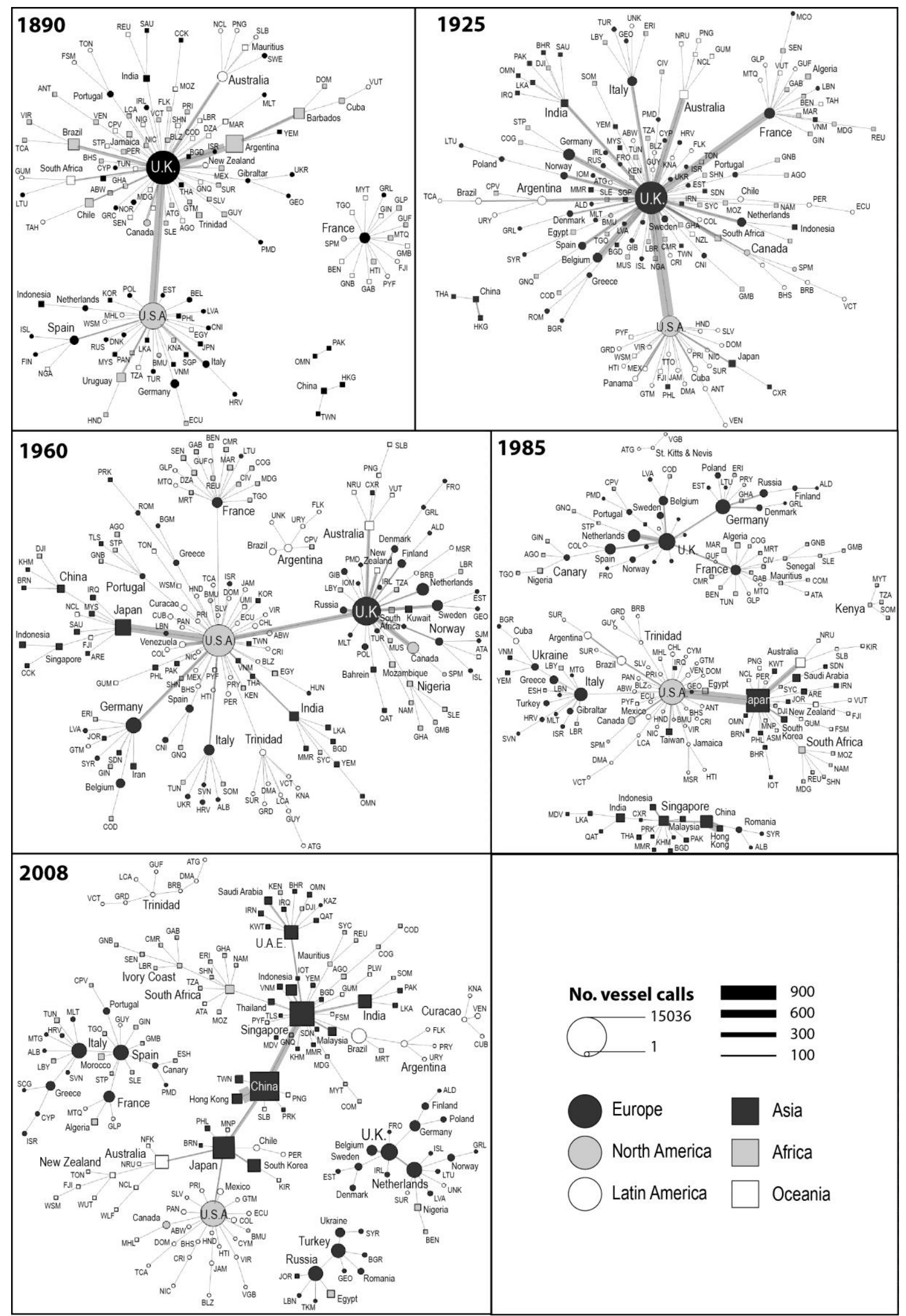

Figure 2 : Flux maritimes dominants entre pays du monde, 1890-2008 